\title{
Strain effect in surface acoustic wave elements with a piezoelectric acoustic line and sensors based on this effect
}

\author{
Ya.I. Lepikh \\ Scientific \& Technical Center "Phonon", Special Design \& Technological Bureau "Element", 29, prospect Acad. Glushko, Odessa, \\ 270104, Ukraine
}

\begin{abstract}
The results of investigations of strain effect in surface acoustic wave (SAW) elements with a piezoelectric ceramic (PC) acoustic line are presented. The choice of PC material for acoustic line is substantiated. Some recommendations concerning formation of interdigital transducer structures of the SAW element with PC acoustic line are given. For acoustic lines of different thicknesses fixed in cantilever a dependence of sensor responsivity on deforming force load is found. At optimum thickness of the PC acoustic line the sensor responsivity of $70 \mathrm{~Hz} / \mathrm{N}$ is realized. The results of this work are compared to those obtained for single-crystal acoustic lines by other authors. A design of a pressure sensor with a strain-sensitive SAW element is presented, and some advantages of such sensors are discussed.
\end{abstract}

Keywords: strain effect, surface acoustic waves, piezoelectric ceramic, pressure sensor.

Paper received 27.05.99; revised manuscript received 15.11.99; accepted for publication 04.01.00.

\section{Introduction}

A great number of papers (see, e.g., [1-5]) deal with the strain effect, that is, changes in the surface acoustic wave (SAW) characteristics due to acoustic line deformation. This problem is of interest since it seems promising for the development of a novel class of SAW measuring transducers for sensors of various physical quantities, such as force, pressure, acceleration, displacement, angular changes, etc. These sensors may demonstrate high metrological performances and be made smart rather easily. The efforts of both researchers and developers in this area, however, are focused mainly on acoustic lines made of single-crystalline piezoelectric materials. Among them are differently cut quartz $[1,2,5]$ and some layered structures, namely, piezoelectric $\mathrm{ZnO}$ film/fused quartz or $\mathrm{ZnO} /$ silicon [3, 4].

Structurally such transducers are made as a freely supported beam, cantilever or membrane. On their working surface the interdigital transducer (IDT) structures are formed using thin-film techniques. These SAW IDT structures serve as resonators or delay lines. This fact alone makes no barrier when following the path from a prototype to commercial instruments. There exist, however, a number of purely physical reasons that present serious obstacles at this path. This results mainly from the fact that, due to anisotropy of single-crystalline acoustic lines, the strains in them do not provide a pure mode of elastic vibrations (in our case, Rayleigh waves). This fact, in particular, makes more difficult solving the problem how to determine a legitimate signal that corresponds to the measured physical quantity. Besides, the electrophysical parameters (low electromechanical coupling) of the acoustic lines used do not enable one to get high sensor responsivity and wide dynamic range that could provide some advantages over the analogs based on other physical principles.

Here we present the results of our investigations of strain effect in SAW elements with a piezoelectric ceramic (PC) acoustic line, as well as development of sensors based on this effect.

\section{Experimental procedure}

In recent years many efficient PC materials have been offered [6-8]. They found extensive applications in microelectronics and instrument engineering [9-11]. One of their features is a rather wide range of their electrophysical parameters. This fact makes the material choice a very important stage when developing a specific product. 
Ya.I. Lepikh: Strain effect in surface acoustic wave elements with...

We investigated a number of PC materials belonging to the lead zirconate-titanate (LZT) system. Generally the main criteria when choosing PC are as follows: high efficiency of mechanical load to electric signal conversion; high temperature, as well as long-term, stability of electrophysical parameters; high Q-factor; high mechanical strength. When SAWs are used, then some additional requirements are placed upon a PC acoustic line. They refer to the value of insertion losses that depend mainly on operating frequency, porosity (granularity) of the material used, working surface finish class and permittivity. The latter determines the capacitive compound of the IDT impedance. Some approaches to the choice of PC materials with an optimum (for definite technical requirements) set of electrophysical parameters, as well as IDT structures, are discussed in $[12,13]$.

The design of the studied PC strain transducer with IDTs on one of its faces is diagrammed in Fig.1. A PC plate (measured $20 \times 7 \mathrm{~mm}^{2}$ ) was fixed in cantilever with a special fixture. Thicknesses of the plates used varied between 0.5 and $1.5 \mathrm{~mm}$. A straining force load whose value, $F$, could be changed was applied to the opposite end of the plate. Two IDTs were placed at the working surface. Contrary to the known design solutions, they formed the amplitude-frequency response of a narrow-band filter whose central frequency, $f_{0}$, was $10 \mathrm{MHz}$. The IDTs have been thinned out with a step of $3 \lambda$, where $\lambda$ is the wavelength [9]. Such a solution offers a number of advantages. In particular, it provides an optimum IDT impedance value, as well as sufficient signal selectivity, while the SAW element is characterized with high manufacturability. The SAW filter in the measuring circuit served as a frequency-driving element in the feedback path of a broadband amplifier. The sensor operating frequency has been chosen so as to provide efficient signal processing using an oscillator circuit, as well as make the technological requirements to IDT fabrication accuracy less stringent. An operating frequency increase (being efficient if microprocessor facilities are used in a sensor) requires a corresponding decrease in both IDT electrodes

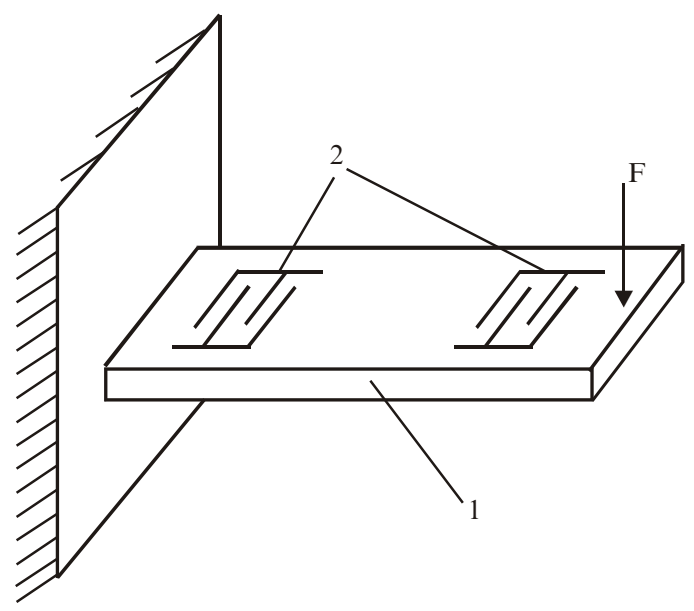

Fig. 1. A diagram showing the design of a strain transducer with SAW element: 1 - PC acoustic line; 2 - IDT. width and spacing, thus meaning that the technological requirements become more severe. Besides, such an operating frequency increase results in an insertion loss rise for a SAW element with a PC acoustic line [14].

It should be noted that the type of PC plate fixation in cantilever used by us was determined by both the design and technological considerations, as well as an experience gained. This enabled us to take into account the specific technical requirements for the sensor used.

The amplitude (dynamic) range depends on the mechanical $Q$ factor, $Q_{\mathrm{M}}$, of the material used. For different makes of PC material $Q_{\mathrm{M}}$ varies from 680 (PZT-2, produced by Murata, Japan) to 4500 (PKR-52, produced at Rostov-on-Don Institute of Physics, Russia).

\section{Experimental results and discussion}

Strain effect has been estimated from the SAW filter central frequency change, $\Delta f=f-f_{0}$, with the force load applied to the free end of the PC plate with IDT structure that was fixed in cantilever. In [3] a theoretical expression has been derived for the relative frequency change as a function of straining force load $F$. Being applied in our case when temperature gradients along the three coordinate axes were assumed to be zero and the oscillator circuit was used for the SAW element connection, this expression takes the following form:

$\left(\frac{\Delta f}{f_{0}}\right)^{S_{1} S_{2}} \approx \sigma_{2 a v}^{S_{1} S_{2}}\left(F_{i}\right)\left(\frac{k}{2 V_{0} \rho_{0}}+\frac{1-2 \mu_{0}}{2 E_{0}}-\frac{1}{E_{0}}\right)+\frac{1}{2} \alpha_{T} T$.

Here $\sigma_{2 a v}^{S_{1} S_{2}}\left(F_{i}\right)=\frac{1}{L} \int_{L} \sigma_{2}^{S_{1} S_{2}}\left(\zeta, F_{i}\right) d \zeta$ is the mechanical stress averaged over the plate length $L$ (i.e., SAW path); $S_{1}\left(S_{2}\right)$ denotes the upper (lower) surface of the strained layer; $k=k / E_{0}$ is the material nonlinearity coefficient (found experimentally); $V_{0}$ is the SAW velocity in the unstrained material; $\rho_{0}$ is the unstrained layer density; $E_{0}$ and $\mu_{0}$ are Young's modulus and Poisson's ratio, respectively; $\alpha_{T}$ is the coefficient of linear expansion of the material; $T$ is the temperature; $\sigma_{2}^{S_{1} S_{2}}$ is the experimentally found strain (assumed to be linear).

Shown in Fig. 2 are the experimental frequency change $v s$ straining load curves for different PC acoustic line thicknesses. The material used was made of TsTS-42-1 make. It demonstrated a rather good (for usual sensor operating conditions) set of parameters, namely: the electromechanical coupling coefficient $K_{p}=0.42$; relative permittivity $\varepsilon_{33}^{T} / \varepsilon=370 \pm 40$; the dielectric losses were characterized by $\operatorname{tg} \delta<0.006$; the mechanical $Q$ factor, $Q_{\mathrm{M}}$, was over 1200; the Curie temperature $T_{c}=603 \mathrm{~K}$. The force load varied from 0 to $0.6 \mathrm{~N}$. One can see that the experimental $\Delta f$ $(F)$ curves have rather long linear portions. This makes a qualitative difference when a comparison is made with analogous curves taken for differently cut quartz [2] and a range 
Ya.I. Lepikh: Strain effect in surface acoustic wave elements with...

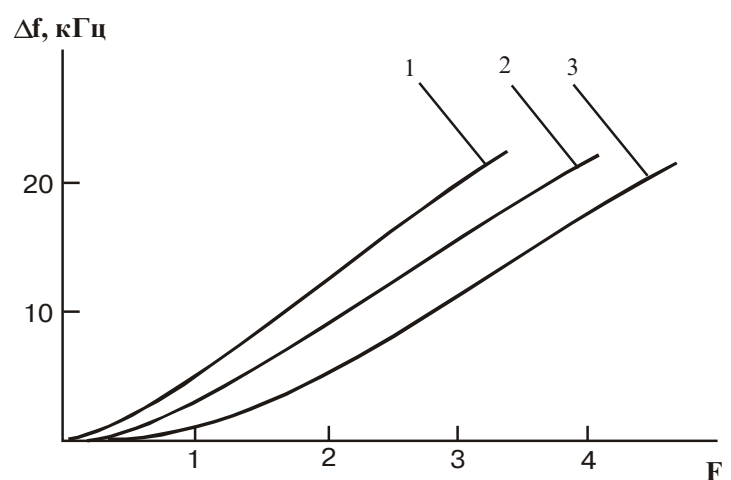

Fig. 2. Sensor frequency change, $\Delta f$, vs strain force load, $F$, curves taken at PC plate thickness of 0.5 (1), 1.0 (2) and $1.5 \mathrm{~mm}$ (3).

of orientations of SAW propagation: the latter curves are essentially nonlinear. Presence of a small nonlinear portion at low load values (see Fig. 2) resembles a residual strain. The sensor time of response at the linear portion of the strain element sensitivity is $1-2 \mathrm{~s}$. The complete resetting time ( $\Delta f=0$ at $F=0$ ) is from 1 to 3 min., depending on the plate thickness. A decrease in PC plate thickness reduces the nonlinear portion and increases both responsivity and operating speed of sensor. But the sensor reliability drops because an admissible (up to deterioration) number of loading cycles goes down in this case. For plates of bigger thicknesses the responsivity drops, and both the nonlinear portion and time of response grow. At PC plate thickness of $1 \mathrm{~mm}$ the sensor responsivity is $70 \mathrm{~Hz} / \mathrm{N}$ and both time of response and reliability are good. This thickness value seems to be optimum.

It should be noted that our sensor has one more advantage. One can control its responsivity in a very simple way, namely, by changing the PC plate sizes.

Basing on the strain sensitive SAW element studied, we have fabricated a pressure sensor of oscillator type with temperature compensation. Its skeleton diagram is given in Fig.3. A nonlinear portion on the strain responsivity has been cut off using a special circuit in the sensor measuring section. The mechanical part of the construction provided such transformation of the force load on the sensitive element that the plate was strained far from its ultimate strength.

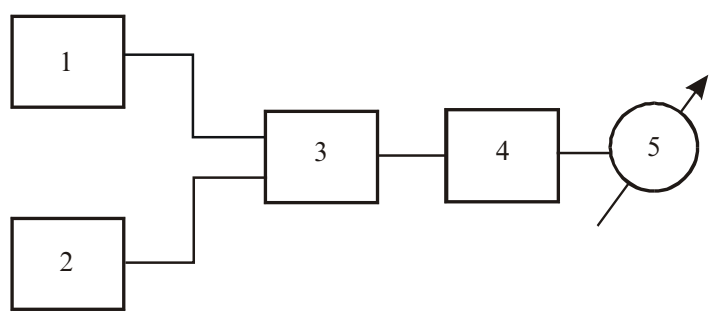

Fig. 3. A schematic diagram of a pressure sensor: 1 - oscillator with a strain transducer; 2 - reference oscillator; 3 - mixer; 4 low-pass filter; 5 - meter.

\section{Conclusions}

Basing on strain effect and using SAW elements, one can fabricate not only pressure sensors but also those of other physical quantities (displacement, angular changes, temperature, etc.). To this end common transducers are to be used that convert the corresponding quantities to strain.

Use of piezoelectric ceramic in our sensor is a factor that gives it one more advantage over its analogs, namely, its low cost. And this may be decisive to win competition existing in this area of instrument engineering.

\section{References}

1. D.V.Shick, H.F.Tierstein, An analysis of the in plane acceleration sensitivity of ST0-cut quartz surface wave resonators with the substrate extending beyond the supports, pp. 230238, in Proc. 42 ${ }^{\text {nd }}$ Ann. Freq. Contr. Symp., Baltimore, Md. 1988, New York (1987).

2. Yu.V.Gulyaev, V.M.Koleshko, Yu,V,Meshkov, Strain effect in SAW structures with a single-crystalline quartz acoustic line (in Russian) // Radiotekhnika i Elektronika 33(11), pp.2448-2451 (1988).

3. I.M.Grankin, V.K.Lopushenko, V.P.Pogrebnyak, N.G.Chernyak, Measuring information converters based on layered structures (in Russian), pp. 182-183, in Proc. Conf. Acoustoelectronic Devices for Information Processing, Moscow (1988).

4. V.I.Anisimkin, E.Verona, G.Socino, Sensors based on SAW in $\mathrm{ZnO} / \mathrm{Si}$ layered structures (in Russian) // Zhurn. Tekhn. Fiz. 58(10), pp. 2051-2054 (1988).

5. Z.U.Tilmann, Strain sensor with commercial SAWR // Sensors and Actuators 17(1-2), pp. 235-239 (1989).

6. P.Duran, F.Lozano, F.Capel, C.Moure, Lange electromechanical anisotropic modified lead titanate ceramics. P.I.Processing. I. // Mater. Sci. 23(12), pp. $4463-4469$ (1988).

7. A.Ya.Dantsiger, L.A.Reznichenko, S.I.Dudkina et al., Ways to prepare highly efficient materials for piezoelectronic devices (in Russian), pp.35-45, in Fundamental Problems of Piezoelectronics. Proc. Intern. Sci. \& Pract. Conf. «Piezoengineering-95”, Kniga Publ., Rostov-on-Don (1995).

8. V.V.Klimov, O.S.Didkovskaya, G.E.Savenkova et al., Donetsk piezoelectric ceramic (in Russian), pp. 59-65, in Fundamental Problems of Piezoelectronics. Proc. Intern. Sci. \& Pract. Conf. «Piezoengineering-95», Kniga Publ., Rostovon-Don (1995).

9. Ya.I.Lepikh, Application of the LZT system ceramic in SAW devices (in Russian) // Elektronnaya Tekhnika. Ser.10. Mikroelektronnye Ustroistva No.5, pp. 48-50 (1988).

10. Ya. Lepikh, Studies of pezoceramic materials for acoustoelectronics, p.69, in Electronics Ceramics Production and Properties. Abstr. Intern. Conf., Ed. A.Krumins, Latvijas Universitate, Riga (1990).

11. V.P.Sakhnenko, From solid state physics to piezoelectronics (in Russian), pp. 5-12, in Fundamental Problems of Piezoelectronics. Proc. Intern. Sci. \& Pract. Conf. «Piezoengineering-95», Kniga Publ., Rostov-on-Don (1995).

12. Ya.I.Lepikh, Yu.V.Gulyaev, A.N.Kalashnikov, Analytical investigation of SAW filters characteristics (in Russian) // Radiotekhnika i Elektronika 33(11), pp. 2395-2399 (1988).

13. Ya.I.Lepikh, An experience of ceramics application in SAW devices and technical requirements to it (in Russian), p. 63, in Ceramics in Economics. Abstr. Sci. \& Techn. Conf., Moscow (1993).

14. Ya.I.Lepikh, S.P.Protopopov, Investigation of the frequency dependence of insertion losses in SAW devices (in Russian) // Tekhnika Sredstv Svyazi. Ser. OT. No. 1, pp. 78-83 (1990). 